\title{
ALTERATIONS IN PULMONARY ARTERY FLOW PATTERNS AND SHEAR STRESS DETERMINED WITH THREE-DIMENSIONAL PHASE-CONTRAST MAGNETIC RESONANCE IMAGING IN FONTAN PATIENTS
}

Victoria L. Morgan, $\mathrm{PhD}^{\mathrm{a}, \mathrm{b}}$

Thomas P. Graham, Jr., MD ${ }^{\mathrm{c}}$

Robert J. Roselli, PhD ${ }^{\mathrm{a}}$

Christine H. Lorenz, $\mathrm{PhD}^{\mathrm{d}}$

\begin{abstract}
Objective: This study compares in vivo pulmonary blood flow patterns and shear stresses in patients with either the direct atrium-pulmonary artery connection or the bicaval tunnel connection of the Fontan procedure to those in normal volunteers. Comparisons were made with the use of three-dimensional phase contrast magnetic resonance imaging. Methods: Three-dimensional velocities, flows, and pulmonary artery cross-sectional areas were measured in both pulmonary arteries of each subject. Axial, circumferential, and radial shear stresses were calculated with the use of velocities and estimates of viscosity. Results: The axial velocities were not significantly different between subject groups. However, the flows and cross-sectional areas were higher in the normal group than in the two patient groups in both pulmonary arteries. The group with the bicaval connection had circular swirling in the cross section of both pulmonary arteries, causing higher shear stresses than in the controls. The disorder caused by the connection of the atrium to the pulmonary artery caused an increase in some shear stresses over the controls, but not higher than those found in the group having a bicaval tunnel. Conclusions: We found that pulmonary flow was equally reduced compared with normal flow in both patient groups. This reduction in flow can be attributed in part to the reduced size of the pulmonary arteries in both patient groups without change in axial velocity. We also found higher shear stress acting on the wall of the vessels in the patients having a bicaval tunnel, which may alter endothelial function and affect the longevity of the repair. ( $J$ Thorac Cardiovasc Surg 1998;116:294-304)
\end{abstract}

$\mathrm{C}$ omplex congenital heart defects such as tricuspid atresia and single ventricle frequently are associated with an obstruction to the pathway for

From the Department of Biomedical Engineering, ${ }^{a}$ the Department of Radiology and Radiological Sciences, ${ }^{\mathrm{b}}$ and the Department of Pediatric Cardiology, ${ }^{\mathrm{c}}$ Vanderbilt University, Nashville, Tenn., and the Cardiovascular Division, Barnes-Jewish Hospital at Washington University Medical Center, ${ }^{\text {d }}$ St. Louis, Mo.

This work was partially supported by the Department of Health and Human Services Public Health Service Grant HL 07411-16 and the Patricia Roberts Harris Fellowship (V.L.M.).

Received for publication June 16, 1997; revisions requested August 8, 1997; revisions received March 5, 1998; accepted for publication March 25, 1998.

Address for reprints: Christine H. Lorenz, PhD, Center for Cardiovascular Magnetic Resonance, Cardiovascular Division, BarnesJewish Hospital at Washington University Medical Center, 216 South Kingshighway Blvd., St. Louis, MO 63110.

Copyright (C) 1998 by Mosby, Inc.

0022-5223/98 \$5.00+ $0 \quad \mathbf{1 2 / 1 / 9 0 6 6 9}$ deoxygenated blood to reach the lungs. In 1971, Fontan performed the first successful procedure to use direct right atrial-pulmonary artery connection to bypass this obstruction. ${ }^{1,2}$ Several modifications to the Fontan procedure have been used, including a cavopulmonary connection in which the inferior vena cava is connected to the right pulmonary artery (RPA) via an intraatrial tunnel and the superior vena cava is connected to the RPA as well.

We have developed an in vivo approach that is capable of determining shear stress using threedimensional phase contrast magnetic resonance imaging (MRI). ${ }^{3-5}$ This technique uses the three-dimensional velocities measured with MRI in addition to viscosity to calculate the shear stress along the vessel wall at different times throughout the cardiac cycle. Therefore the purpose of this study was to use the three-dimensional blood velocity characteristics to calculate and compare three types of shear stress 

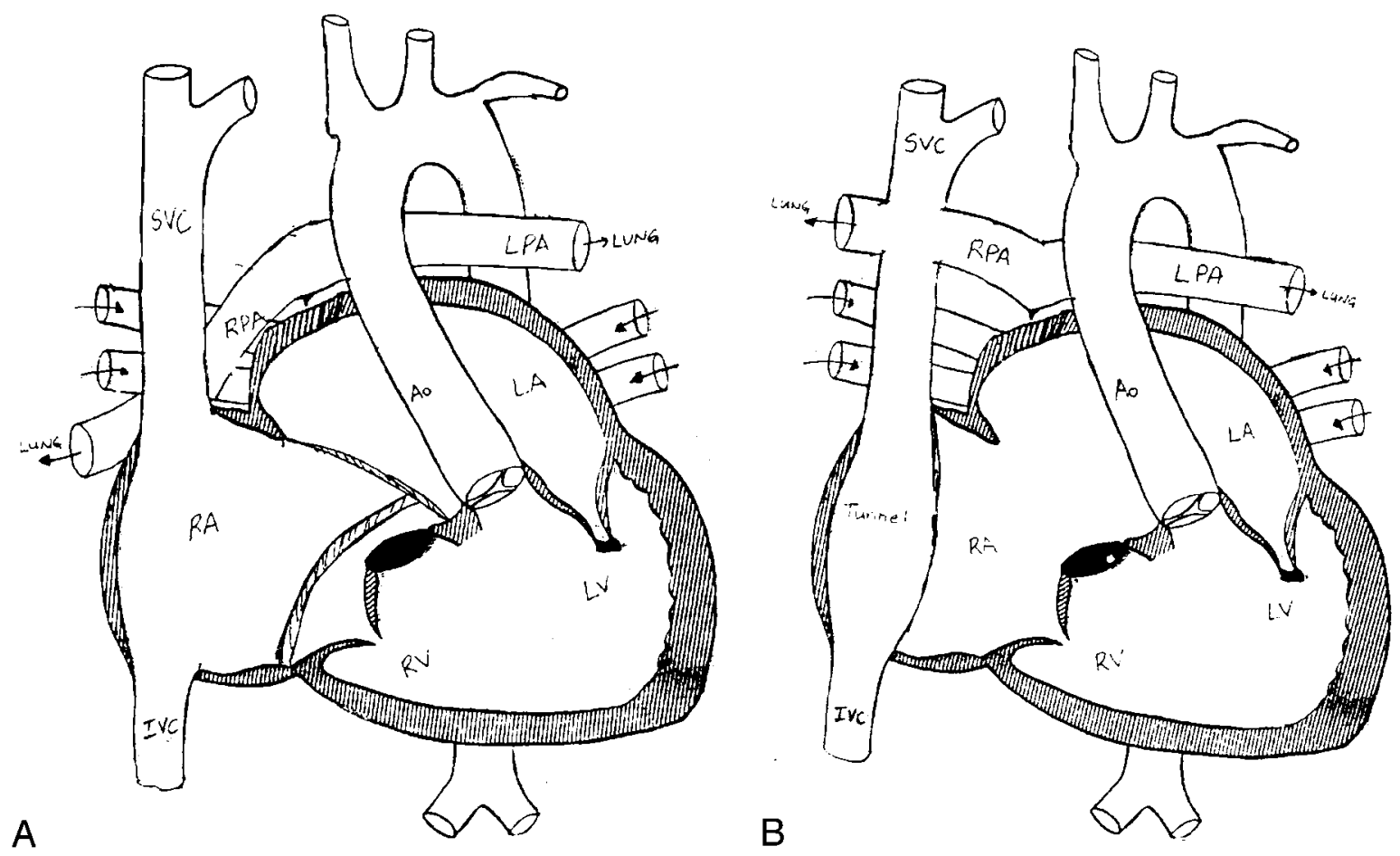

Fig. 1. Diagrams showing the Fontan repair in the RA group (A) and the BC group (B). SVC, Superior vena cava; $I V C$, inferior vena cava; $R A$, right atrium; $R P A$, right pulmonary artery; $R V$, right ventricle; $L V$, left ventricle; $L A$, left atrium; $L P A$, left pulmonary artery; $A o$, aorta.

Table I. Subject information

\begin{tabular}{lccccc}
\hline & Sex & Age $(y r)$ & Years postop & BSA $\left(\mathrm{m}^{2}\right)$ & Diagnosis \\
\hline Control 1 & M & 22.8 & NA & 1.91 & N/A \\
Control 2 & M & 18.4 & NA & 1.87 & N/A \\
Control 3 & M & 19.3 & NA & 2.00 & N/A N/A \\
Control 4 & F & 19.1 & NA & 1.67 & N/A \\
Control 5 & F & 20.3 & NA & 2.02 & N/A \\
Control 6 & F & 25.6 & NA & 1.49 & Tricuspid atresia \\
Control 7 & M & 31.5 & NA & 2.11 & SV, PS, S/P Glenn shunt \\
RA 1 & M & 4.0 & 0.9 & 0.68 & Double-inlet LV, TGA, PS \\
RA 2 & F & 13.5 & 6.1 & 1.66 & SV, TGA, PS \\
RA 3 & M & 24.3 & 10.1 & 1.84 & Double-outlet RV, L-TGA \\
RA 4 & M & 27.3 & 8.3 & 2.14 & Tricuspid atresia, L-TGA \\
RA 5 & M & 23.6 & 11.9 & 1.64 & Double-inlet LV, PS \\
RA 6 & F & 8.9 & 2.7 & 1.01 & Tricuspid, pulmonary atresia \\
BC 1 & M & 29.2 & 4.1 & 1.77 & SV, pulmonary atresia \\
BC 2 & F & 16.2 & 1.1 & 1.70 & Double-inlet LV, L-TGA \\
BC 3 & M & 11.4 & 1.5 & 1.25 & Pulmonary atresia, SV, TGA \\
BC 4 & M & 5.9 & 4.1 & 0.83 & 1.35
\end{tabular}

Years postop, Years since Fontan operation; $B S A$, body surface area; $N A$, not applicable; $S V$, single ventricle; $P S$, pulmonic stenosis, $S / P$, status post; $L V$, left ventricle; $T G A$, transposition of the great arteries.

in the pulmonary arteries of normal volunteers with those in two groups of patients undergoing the Fontan operation with different types of connections. The three types of shear stress studied were the axial shear stress, the circumferential shear stress, and the radial shear stress, which act in opposition to the axial, circumferential, and radial pressure gradients, respectively. 

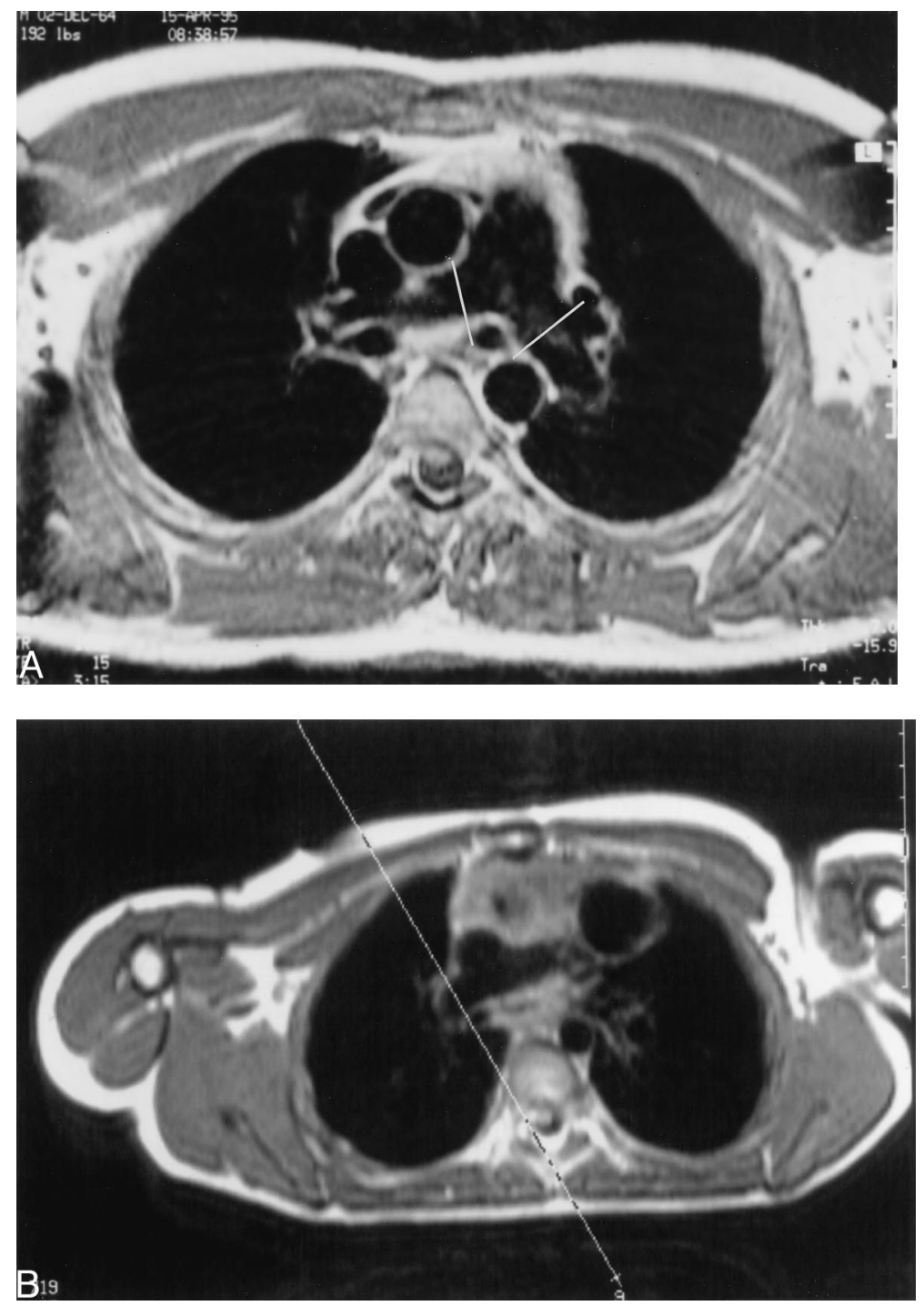

Fig. 2. T1-weighted transverse image through the pulmonary arteries. The main pulmonary artery bifurcates into the LPA and RPA. The velocity encoded cine imaging slice positions through (A) both vessels in the control group and (B) the RPA in the BC group is shown.

\section{Patients and methods}

Study population. Three-dimensional phase contrast cine MRI was used to determine pulmonary blood flow in normal controls and in patients who had undergone the Fontan procedure 1 to 12 years previously. The patients included six subjects who had a right atrial-pulmonary artery connection as shown in Fig. 1, $A$ (RA group, 2 female patients, 4 male patients, aged 4 to 27 years, 1 with a right atrial conduit) and five subjects who had a bicaval tunnel (BC group, 4 male patients, 1 female patient, aged 5 to 29 years, 3 with fenestrations). In the $\mathrm{BC}$ group, the junctions of the tunnel with the RPA formed a T-junction and were not offset (Fig. 1, B). In the RA group, the four patients without tricuspid atresia had patch closure of the tricuspid valve. All patients were free of thrombotic complications by history and echocardiography. The control group consisted of seven subjects (4 male patients, 3 female patients, aged 18 to 31 years). Table I describes the study population. Informed consent as directed by the Vanderbilt Institutional Review Board was obtained for each subject.

Imaging. The MRI signal is composed of a signal magnitude, which is normally displayed in conventional MRI, and phase. Phase contrast MRI uses a phase shift induced by moving tissue to create images of velocity. ${ }^{3-5}$ Phase images are then created in which the intensity of each pixel is proportional to the average velocity in the imaged voxel in one direction ( $\mathrm{x}, \mathrm{y}$, or $\mathrm{z})$.

The images were acquired using the body coil of a $1.5 \mathrm{~T}$ Siemens Magnetom SP 4000 scanner (Siemens Medical 
Systems, Inc., Iselin, N.J.). T1-weighted spin echo transverse images, $7 \mathrm{~mm}$ thick, were acquired from the top of the aortic arch to the apex of the heart. Velocity-encoded cine imaging was performed in the RPA and LPA by means of the following pulse sequence parameters: encoded velocity $(\mathrm{z})=$ $2000 \mathrm{~mm} / \mathrm{sec}$, encoded velocity $(\mathrm{xy})=750 \mathrm{~mm} / \mathrm{sec}$, field of view $=300 \mathrm{~mm}$, slice thickness $=10 \mathrm{~mm}$, matrix size $=$ $256 \times 256$, echo time $=10 \mathrm{msec}$, repetition time $=66 \mathrm{msec}$, number of averaged acquisitions $=1$, and number of phase encoding lines per segment $=3$. The cines were performed perpendicular to each pulmonary artery immediately distal to the bifurcation of the main pulmonary artery, as shown in Fig. 2, $A$. In the patients who had the Fontan operations, the RPA velocity encoded imaging slices were placed distal to the bicaval tunnel connection (Fig. 2,B) or to the right atrial connection.

Data analysis. Velocities in the $x-y$ (in-plane) and $z$ (axial) directions were calculated for each pixel in the vessel cross section and averaged with the use of methods developed previously. ${ }^{6}$ The data were plotted versus percent R-R interval. These curves were compared between subject groups by means of the multifactorial analysis of variance (ANOVA) test. Where statistical significance was determined, the data were compared by the one-way ANOVA with a Bonferroni subtest.

Distensibility was calculated as difference between the maximum and minimum cross-sectional areas through the cardiac cycle divided by the maximum cross-sectional area through the cardiac cycle. The flow in milliliters per second at each phase of the cardiac cycle was calculated as the average axial velocity multiplied by the cross-sectional area of the vessel.

Shear stress calculations. The axial shear stress, $\tau_{\text {rz }}$, circumferential shear stress, $\tau_{\mathrm{r} \theta}$, and radial shear stress, $\tau_{\text {rr }}$, were measured at each pixel near the wall of the vessel by means of equations 1 through $3^{7}$ :

$$
\begin{gathered}
\tau_{\mathrm{rz}}=-\mu\left[\frac{\partial \nu_{\mathrm{z}}}{\partial \mathrm{r}}+\frac{\partial \nu_{\mathrm{r}}}{\mathrm{dz}}\right] \\
\tau_{\mathrm{r} \theta}=-\mu\left[\mathrm{r} \frac{\partial}{\partial \mathrm{r}}\left(\frac{\nu_{\theta}}{\mathrm{r}}\right)+\frac{1 \partial \nu_{\mathrm{r}}}{\mathrm{r} \partial \theta}\right] \\
\tau_{\mathrm{rr}}=-\mu\left[2 \frac{\partial \nu_{\mathrm{r}}}{\partial \mathrm{r}}-\frac{2}{3}(\nabla \cdot \nu)\right]
\end{gathered}
$$

where $\mu$ is the coefficient of viscosity of the blood ( 0.003 $\mathrm{gm} / \mathrm{mm} \cdot \mathrm{sec}), \nu_{\mathrm{z}}$ is the axial velocity, $\nu_{\mathrm{r}}$ is the radial velocity, $\mathrm{v}_{\theta}$ is the circumferential velocity, $\mathrm{r}$ is the distance between the pixel and the center of the vessel, and $\theta$ is the angle between the horizontal plane through the center of the cross section and the radius of the pixel, as shown in Fig. 3. The values for $\nu_{\mathrm{r}}$ and $\nu_{\theta}$ can be computed from $\nu_{\mathrm{x}}$ and $\nu_{\mathrm{y}}$ by means of rotational matrices. ${ }^{8}$ The pixels at the wall of the vessel were chosen as those that outlined the region of interest. The pixels near the wall of the vessel were then determined as those pixels directly adjacent to the wall pixels.

The expressions in the form $\partial \nu \cdot \partial \mathrm{r}$ were calculated for each pixel, $\mathrm{p}_{\mathrm{i}}$, by determining the neighboring pixel closest to the center, $\mathrm{p}_{\mathrm{i}-1}$, and calculating:
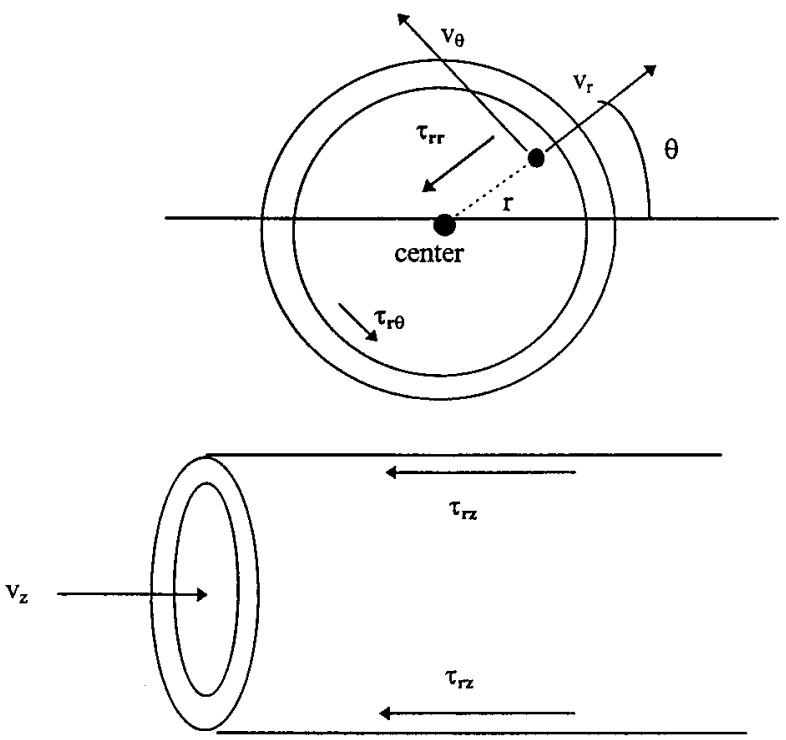

Fig. 3. A section of the vessel showing a single pixel and its axial velocity $\left(v_{z}\right)$, circumferential velocity $\left(v_{\theta}\right)$, radial velocity $\left(v_{r}\right)$, the axial shear stress $\left(\tau_{r z}\right)$, the circumferential shear stress $\left(\tau_{r \theta}\right)$, and the radial shear stress $\left(\tau_{r r}\right)$.

$$
\frac{\partial \nu}{\partial \mathrm{r}}=\frac{\text { Velocity at } \mathrm{p}_{\mathrm{i}}-\text { Velocity at } \mathrm{p}_{\mathrm{i}-1}}{\text { Radius at } \mathrm{p}_{\mathrm{i}}-\text { Radius at } \mathrm{p}_{\mathrm{i}-1}}
$$

Similarly, the expressions in the form $\partial \nu \cdot \partial \theta$ were calculated using the neighboring pixel in the counterclockwise direction around the vessel wall and its change in angle, $\theta$. The $\partial \nu \cdot{ }_{\mathrm{r}} \partial \mathrm{z}$ term in equation 1 could not be calculated with only one imaging slice in each vessel, but was assumed to be negligible with respect to $\partial \nu \cdot{ }_{z} \partial \mathrm{r}$, especially near the wall. The expression $(\nabla \cdot \nu)$ in equation 3 was assumed to be zero in this case because blood was assumed to be an incompressible, Newtonian fluid.

The absolute value of the shear stress of each pixel at the wall was averaged over the entire wall length, and this mean was plotted versus percent R-R interval. Similarly, the shear stress (without absolute value) and its standard deviation were used to determine relative dispersion (standard deviation/mean) of shear stress along the vessel wall. The absolute value of the axial, circumferential, and radial shear stress over the cardiac cycle was compared between vessels by means of the multifactorial ANOVA test. Where statistical significance was determined, the one-way ANOVA with Bonferroni subtest was used.

\section{Results}

Axial velocity. Fig. 4 shows the flow versus time graphs for each subject group in the LPA and RPA. Notice that the systolic flow peak in the control group is not seen in the patient groups owing to the lack of influence of a right ventricular pumping chamber. Table II shows the results of the axial velocity parameter comparison between subject 

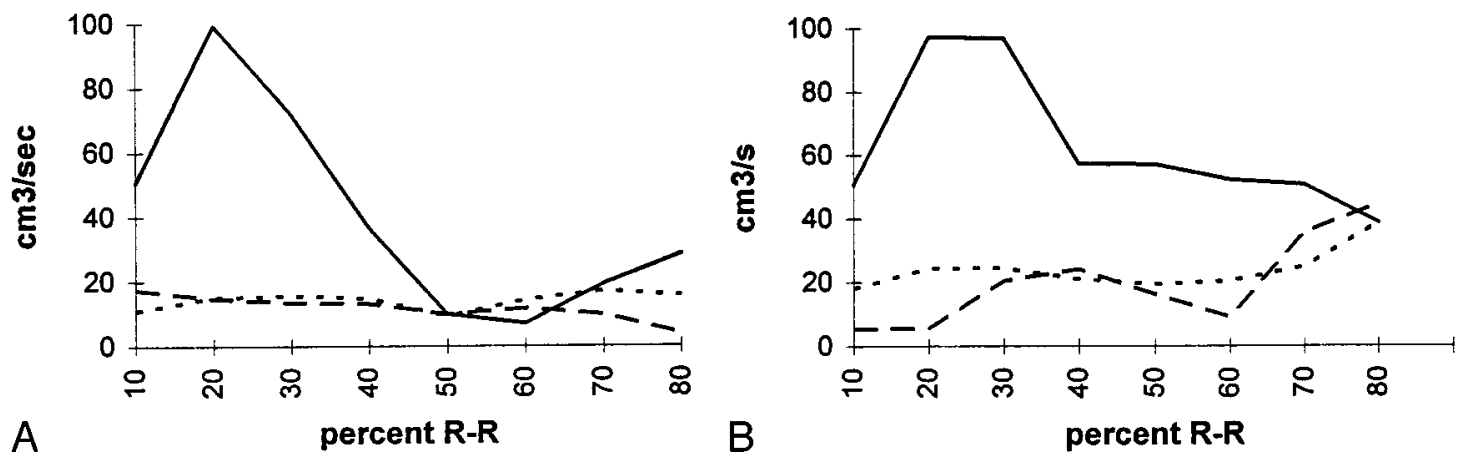

Fig. 4. Flow versus percent RR interval averaged over subject group in the RPA (A) and LPA (B). Control group (solid line), RA group (dotted line), and BC group (dashed line). Standard deviations were similar in all groups, in the range 15 to $30 \mathrm{~cm} / \mathrm{sec}$.

Table II. Blood velocity and flow measurements in the axial direction in the RPA and LPA of all subjects (mean \pm standard deviation)

\begin{tabular}{lccc}
\hline & Control group & RA group & BC group \\
\hline Total flow/BSA $\left(\mathrm{ml} / \mathrm{min} / \mathrm{m}^{2}\right)$ & $2865.7 \pm 460.7^{*}$ & $1430.1 \pm 741.4$ & $1443.2 \pm 748.3$ \\
RPA flow/BSA $\left(\mathrm{ml} / \mathrm{min} / \mathrm{m}^{2}\right)$ & $1144.0 \pm 630.5^{*}$ & $660.1 \pm 526.7$ & $698.7 \pm 284.5$ \\
LPA flow/BSA $\left(\mathrm{ml} / \mathrm{min}^{2}\right)$ & $1721.7 \pm 536.7^{*}$ & $769.9 \pm 602.2$ & $744.5 \pm 491.1$ \\
Total PA area/BSA $\left(\mathrm{mm}^{2} / \mathrm{m}^{2}\right)$ & $268 \pm 48^{*}$ & $131 \pm 34$ & $150 \pm 98$ \\
RPA area/BSA $\left(\mathrm{mm}^{2} / \mathrm{m}^{2}\right)$ & $118 \pm 17 \dagger^{\mathrm{RA}}$ & $51 \pm 16$ & $75 \pm 48$ \\
LPA area/BSA $\left(\mathrm{mm}^{2} / \mathrm{m}^{2}\right)$ & $150 \pm 53^{*}$ & $79 \pm 27$ & $75 \pm 50$ \\
RPA distensibility & $0.44 \pm 0.15$ & $0.45 \pm 0.07$ & $0.33 \pm 0.12$ \\
LPA distensibility & $0.34 \pm 0.10$ & $0.41 \pm 0.19$ & $0.37 \pm 0.13$ \\
RPA mean axial velocity $(\mathrm{mm} / \mathrm{sec})$ & $186 \pm 101$ & $231 \pm 101$ & $258 \pm 159$ \\
LPA mean axial velocity $(\mathrm{mm} / \mathrm{sec})$ & $235 \pm 84$ & $195 \pm 176$ & $230 \pm 117$ \\
RPA peak axial velocity $(\mathrm{mm} / \mathrm{sec})$ & $491 \pm 148$ & $447 \pm 180$ & $438 \pm 240$ \\
LPA peak axial velocity $(\mathrm{mm} / \mathrm{sec})$ & $502 \pm 158$ & $423 \pm 262$ & $457 \pm 297$ \\
\hline
\end{tabular}

$B S A$, Body surface area; $R A$, right atrial-pulmonary artery group; $B C$, bicaval tunnel group; $R P A$, right pulmonary artery; $L P A$, left pulmonary artery. *Statistical difference between control group and both RA and BC groups $(p=0.000075$, control vs RA, and $p=0.0000016$, control vs BC, for total flow/BSA; $p=0.027$, control vs RA, and $p=0.009$, control vs $\mathrm{BC}$, for RPA flow/BSA; $p=0.01$, control vs RA, and $p=0.0003$, control vs BC, for LPA flow/BSA; $p=$ 0.002 , control vs RA, and $p=0.015$, control vs $\mathrm{BC}$, for total PA area/BSA; $p=0.01$ control vs RA, and $p=0.009$, control vs BC, for LPA area/BSA.)

$\dagger^{\mathrm{RA}}$ Denotes statistical difference $(p=0.013)$ between control and RA groups.

groups. The mean and peak axial velocities were not statistically different between subject groups. However, the cross-sectional area and, therefore, the flows were higher in the control group than in both patient groups, but the patient groups were not significantly different from each other.

In-plane velocity. Fig. 5 shows the vessel cross section containing two-dimensional in-plane velocity vectors during systole in the RPA of a control subject, an RA subject, and a BC subject. Fig. 6 gives an example of the same information in the LPA. In the LPA, the mean in-plane velocity in the region of interest was higher in the control group than in either the RA or BC groups, but in the RPA, no difference was found.

Shear stress. Fig. 7 shows the mean axial shear stress at the wall of the vessel, $\tau_{\mathrm{rz}}$, averaged over each subject group. In the RPA, the BC group had a higher axial shear stress than the RA and control groups, and the control group and RA group were not statistically different. In the LPA, the BC group showed higher mean axial shear stress than the RA group, and the control group and the RA group showed higher axial shear stress than the control group.

Fig. 8 shows the mean circumferential shear stress at the wall of the vessel, $\tau_{\mathrm{r} \theta}$, averaged over each subject group. In the RPA, both patient groups had higher circumferential shear stress than the control group, but they were not significantly different from each other. In the LPA, the circumferential shear stress was higher in the $\mathrm{BC}$ group than in both the RA group and the control group.

Fig. 9 shows the mean radial shear stress at the wall of the vessel, $\tau_{\mathrm{IT}}$, averaged over each subject 


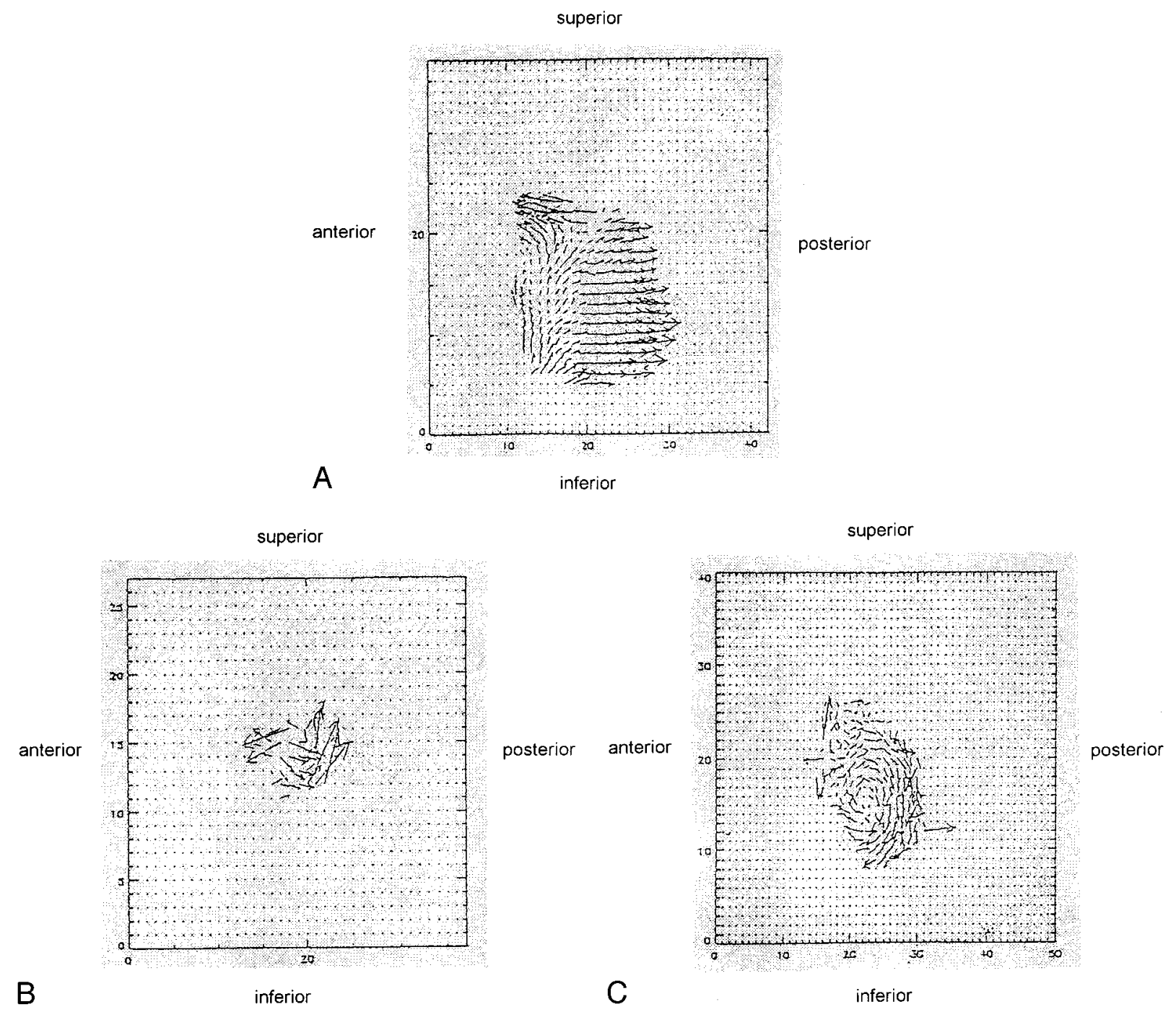

Fig. 5. Two dimensional in-plane blood velocity vectors during systole in the cross section of (A) a normal control subject immediately distal to the main pulmonary artery bifurcation, (B) an RA group subject immediately distal to Fontan connection, and (C) a BC group subject immediately distal to the Fontan connection. The magnitude of the velocity is represented by the length of the vector and the direction of the velocity is represented by the direction of the vector. The axis scale is in pixels at $1.17 \mathrm{~mm} / \mathrm{pixel}$.

group. In the RPA, the $\mathrm{BC}$ group had higher radial shear stress than the control group, but not higher than that of the RA group. However, in the LPA, the $\mathrm{BC}$ group had higher radial shear stress than both the RA group and the control group, but the RA and control groups were not significantly different from each other. All shear stress statistical data are summarized in Table III.

\section{Discussion}

Significance and implications. Various researchers have attempted to compare the energy losses associated with the $\mathrm{RA}$ and $\mathrm{BC}$ connections using models. ${ }^{9,}{ }^{10}$ Using computer modeling, Van Haesdonck and associates ${ }^{10}$ found that the $\mathrm{BC}$ connection had nearly laminar flow, which resulted in lower energy losses than the RA connection. Low, Chew, and $\mathrm{Lee}^{9}$ also calculated lower energy losses in the $\mathrm{BC}$ connection than the RA connection using experimental models. Although three-dimensional models are invaluable for studying the effects of different surgical connections, few methods exist to compare the connections using in vivo measurements. 


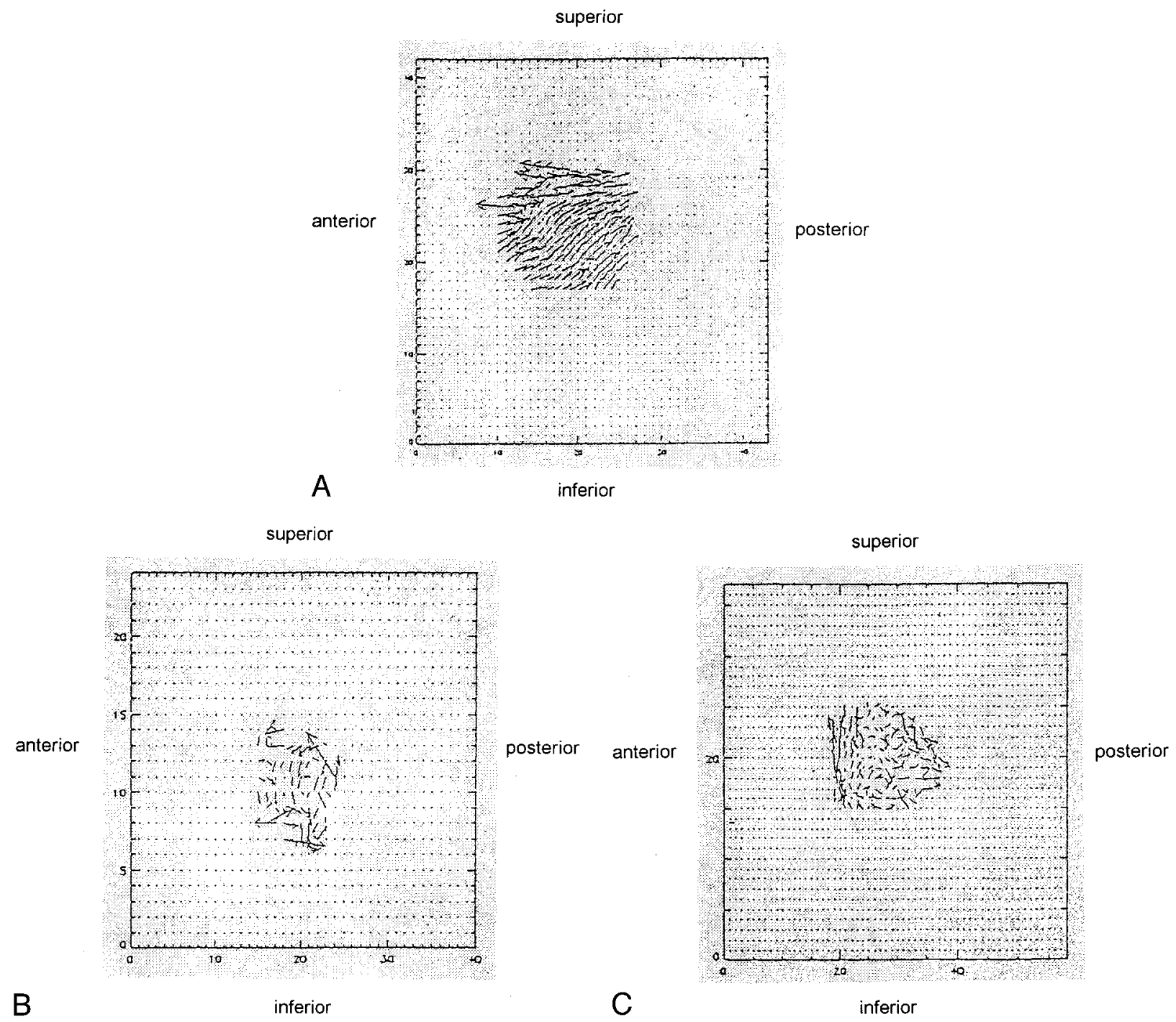

Fig. 6. Two-dimensional in-plane blood velocity vectors during systole in the LPA cross section immediately distal to the main pulmonary artery bifurcation of (A) a normal control subject, (B) an RA group subject, and (C) a BC group subject. The magnitude of the velocity is represented by the length of the vector and the direction of the velocity is represented by the direction of the vector. The axis scale is in pixels at $1.17 \mathrm{~mm} /$ pixel.

The hemodynamic parameters that affect the performance and ultimate longevity of the low-pressure conduits associated with Fontan repairs are not known. Abnormalities of shear stress at the vessel anastomosis represent an excellent candidate for such effects for the following reasons. First, researchers have determined that high shear stresses acting on the vascular endothelium can have a negative effect on both cell function ${ }^{11,12}$ and structure. ${ }^{11,13,14}$ Second, others have found that high shear stresses affect vascular tone by increasing the release of vasodilators and reducing the release of vasoconstrictors by the endothelial cells. ${ }^{11,15-19}$ Last, high shear stress has also been associated with decreased thrombosis. ${ }^{20,21}$ Thus it is critical to understand the role of shear stress after such repairs to interpret conduit performance and examine alternative strategies for repairs based on fundamental principals of fluid mechanics. The technique described here is one noninvasive imaging modality that can provide three-dimensional velocity information to characterize blood flow patterns in vivo. 
Axial velocity. It was found that total pulmonary blood flow normalized to body surface area in the control group was significantly higher than in both patient groups, but that flows in the patient groups were not significantly different from each other. The axial velocities, however, did not differ statistically between subject groups, as seen in Table II, and thus the higher total flow in the controls can be attributed to the higher total pulmonary artery crosssectional area in the control group than the patient groups.

The peak velocities and distensibility data in the two Fontan patient groups were not found to be significantly different from those in the control group. This result is not intuitive, because the flow patterns in the pulmonary arteries of patients who have had the Fontan operation are characterized by very low phasic flow owing to a lack of ventricular pumping chamber. The high standard deviations found in these measurements suggest that definitively concluding that distensibility and peak velocities are not different between groups is beyond the capability of this study. However, increased numbers of subjects or improvements in the MRI techniques may permit better characterization of these parameters.

Pulmonary artery size. The sizes of the RPA and the LPA in the control group fell within ranges reported by Paz, Mohiaddin, and Longmore ${ }^{22}$ for normal pulmonary artery size measured by MRI. The size of the LPA and RPA in the RA and BC groups fell within ranges found by Fogel and coworkers ${ }^{23}$ who used MRI to measure pulmonary artery size throughout stages of the Fontan repair.

Others ${ }^{24-26}$ have studied the size of the pulmonary arteries at autopsy or at takedown of the Fontan repair. All results showed that smaller pulmonary artery size increased the risk of death or takedown. Our results showed that pulmonary artery size as compared with velocity was a dominant factor related to the quantity of pulmonary artery flow, which may be a reason for the relationship between pulmonary artery size and successful outcome of the procedure.

In-plane velocity and shear stress. In the RPA, the mean magnitude of in-plane velocity over the vessel cross section in the control group was not found to be significantly different from that in either the RA or BC group.

In the LPA, however, mean in-plane velocity was significantly higher in the control group than in both the RA and $\mathrm{BC}$ groups. This may be due in part to
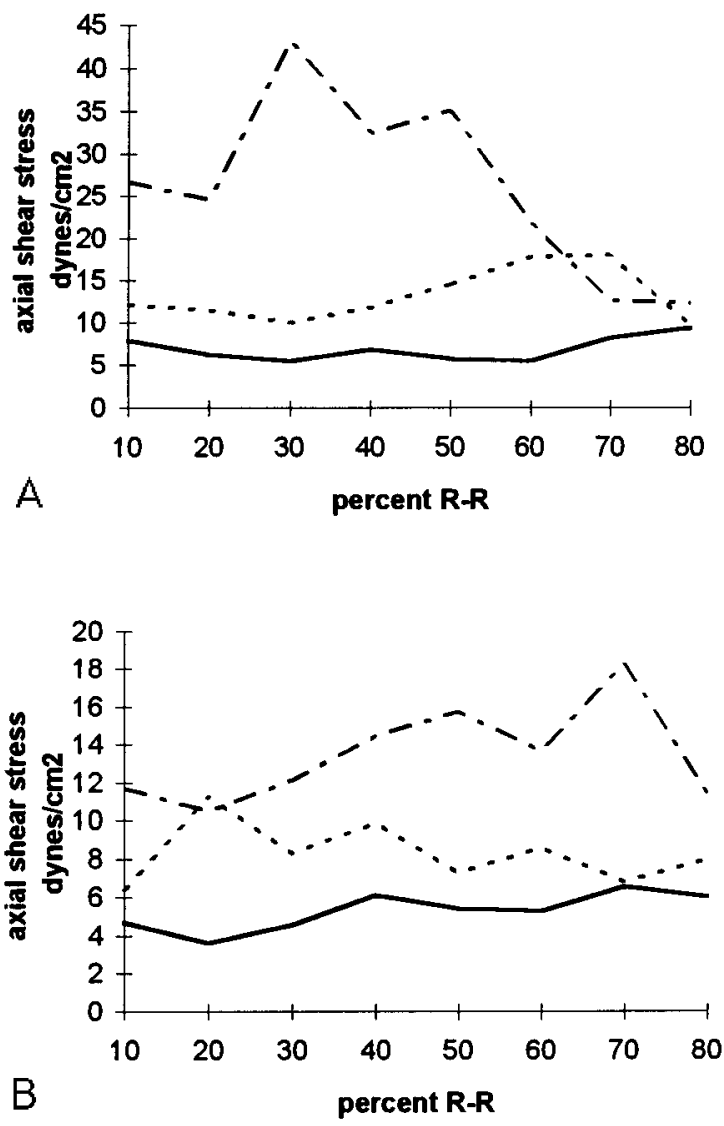

Fig. 7. Axial shear stress near the wall of the vessel averaged over each subject group in the (A) RPA and (B) LPA estimated using three-dimensional phase contrast MRI. Control group (solid line), RA group (dashed line), $\mathrm{BC}$ group (dash/dot/dash line).

the location of the imaging slice. In all groups, the imaging slice was placed immediately distal to the bifurcation of the RPA or LPA. In the controls, the blood flows from the main pulmonary artery, whereas in the patient groups, the blood flows from the Fontan connection in the RPA and has more distance for in-plane velocity variations to stabilize before reaching the imaging slice.

When examining the in-plane velocity directional patterns in the RPA, as shown in Fig. 5, it is evident that the flow pattern from the subject in the BC group (Fig. 5, C) has a circular pattern. In the RPA, the $\mathrm{BC}$ group and the RA group had a significantly higher mean circumferential shear stress over the vessel wall than the control group, indicating that both Fontan connections increase circumferential shear near the connection. In the LPA, further away from the connection, the circumferential shear 

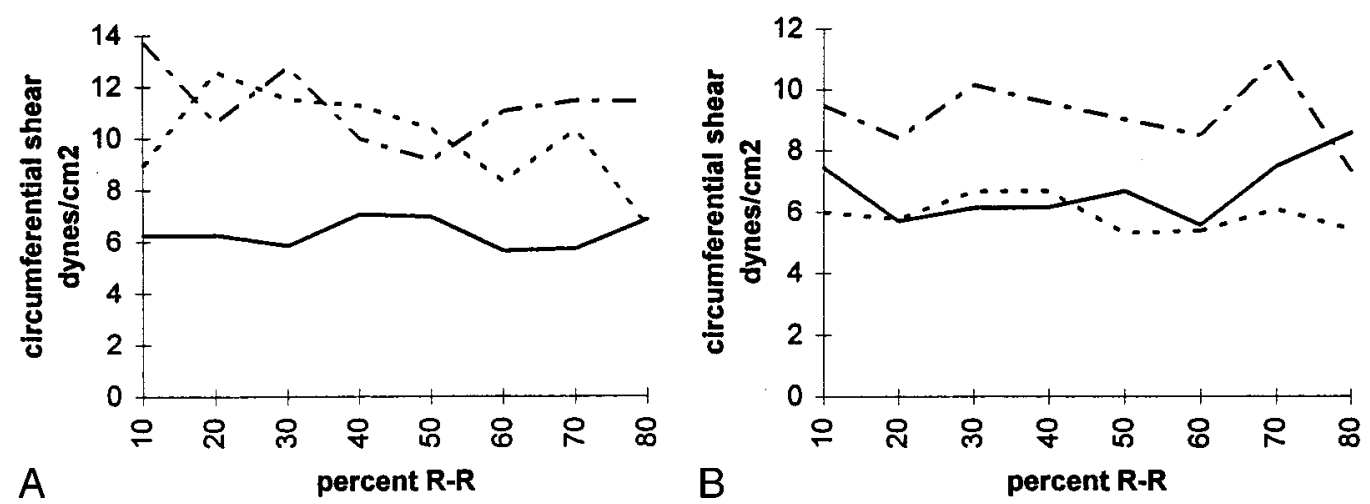

Fig. 8. Circumferential shear stress near the wall of the vessel averaged over each subject group in the (A) RPA and (B) LPA estimated using three-dimensional phase contrast MRI. Control group (solid line), RA group (dashed line), BC group (dash/dot/dash line). Standard deviation of circumferential shear stress was in the range of 120 to 170 dynes $/ \mathrm{cm}^{2}$ in the control group and 53 to 260 dynes $/ \mathrm{cm}^{2}$ in the patient groups.
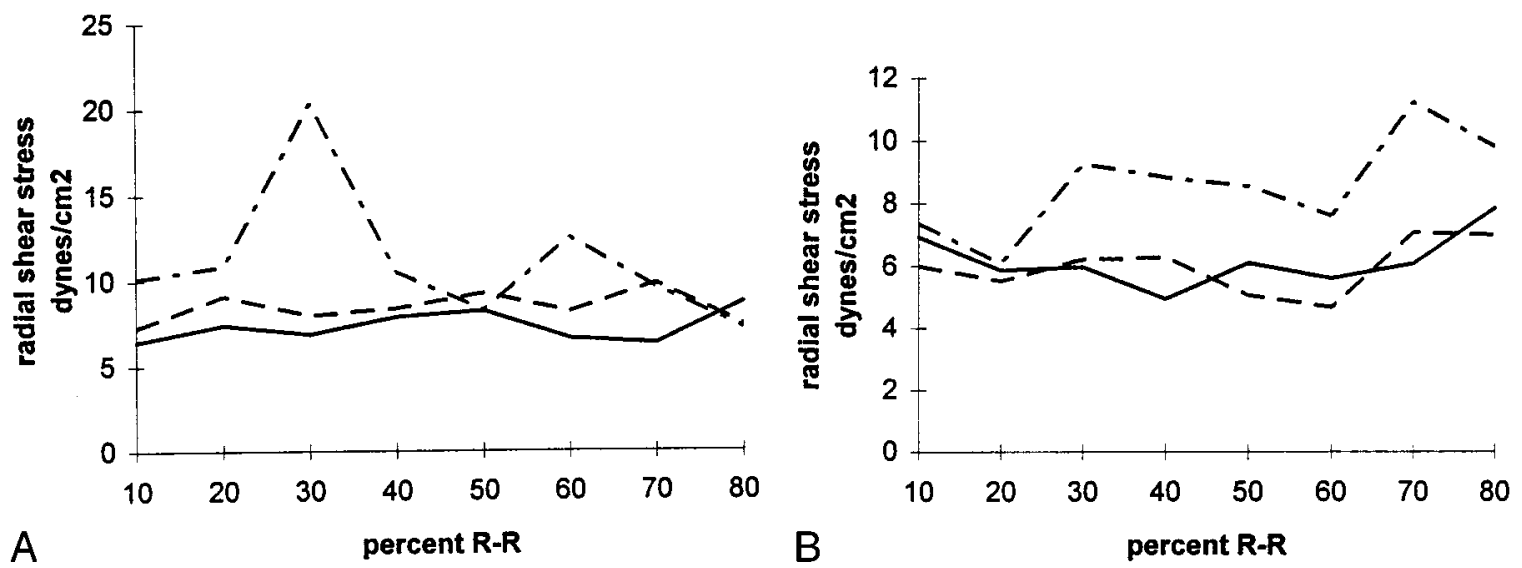

Fig. 9. Radial shear stress near the wall of the vessel averaged over each subject group in the (A) RPA and

(B) LPA estimated using three-dimensional phase contrast MRI. Control group (solid line), RA group (dashed line), BC group (dash/dot/dash line). Standard deviation of radial shear stress was in the range 132 to 138 dynes $/ \mathrm{cm}^{2}$ in the control group and 75 to $302 \mathrm{dynes} / \mathrm{cm}^{2}$ in the patient groups.

Table III. Statistical results from shear stress analysis

\begin{tabular}{lcc}
\hline & $R P A$ & $L P A$ \\
\hline Axial shear stress & $\mathrm{BC}>\mathrm{RA}=\mathrm{C}$ & $\mathrm{BC}=\mathrm{RA}>\mathrm{C}$ \\
Circumferential shear stress & $\mathrm{BC}=\mathrm{RA}>\mathrm{C}$ & $\mathrm{BC}>\mathrm{RA}=\mathrm{C}$ \\
Radial shear stress & $\mathrm{RA}=\mathrm{BC}>\mathrm{C}=\mathrm{RA}$ & $\mathrm{BC}>\mathrm{RA}=\mathrm{C}$ \\
\hline
\end{tabular}

$>$, Significantly greater than $(p<0.05) ;=$, not significantly different. $B S A$, Body surface area; $R A$, right atrial-pulmonary artery group; $B C$, bicaval tunnel group; $R P A$, right pulmonary artery; $L P A$, left pulmonary artery.

stresses were significantly higher in the $\mathrm{BC}$ group and not significantly different between the controls and RA group.

In addition to having higher circumferential shear stresses, the BC group also had higher axial and radial shear stresses. With the swirling pat- tern found in the $\mathrm{BC}$ group, the term $\alpha \nu \cdot{ }_{\mathrm{r}} \alpha \mathrm{Z}$ likely cannot be assumed to be negligible in these subjects. However, even if this term is high in these patients, it would only further increase the axial shear stress.

de Leval and coworkers ${ }^{27}$ found that in their model of a BC connection the energy losses could be reduced by angling the intraatrial tunnel alongside the RPA. This technique was not incorporated in the surgical technique in these patients. Also, Sharma and colleagues $^{28}$ performed power loss measurements in the $\mathrm{BC}$ connection while varying the offset of the superior vena cava toward the LPA. They reported the highest power losses in the zero offset position, which they attributed to "chaotic" flow patterns. The patients with a BC connection in 
this study had a zero offset connection with the superior vena cava angled slightly toward the LPA. Therefore, if the recent surgical recommendations for reduction of energy losses are used, the shear stresses may also be lower than those found in the present study.

Study limitations. Changes in heart rate or patient movement during scanning can blur the images and create errors, because the images are acquired over many heartbeats. In addition, greater statistical significance may have been reached in some parameters if the number of patients in each group had been increased and if their diagnoses had been more uniform

Another important source of variability in this study is the accuracy of the orientation of the imaging axes. In this study, the superior-inferior angulation was small by visual inspection $(<30$ degrees), which would result in errors of in-plane velocities no larger than $13 \%$. We made this tradeoff in protocol time versus velocity uncertainty to reduce total exam time. The position of the imaging slice may have also resulted in decreased measured RPA flow in the patients having the Fontan repairs because of failure to include flow to the right upper lobe due to its proximity to the cavopulmonary anastomosis.

Although other studies have used a higher number of frames for velocity mapping, the actual number of frames necessary to capture the relevant information is dictated by sampling theory. In later studies of pulmonary blood flow we performed at a sampling frequency of $35 \mathrm{~Hz}$ on improved MRI equipment, we performed a frequency analysis of the data. No significant power in the frequency spectrum was seen higher than $5 \mathrm{~Hz}$. Therefore, by the Nyquist criterion, the sampling frequency required to capture this information is 10 $\mathrm{Hz}$. We sampled at a rate of $15 \mathrm{~Hz}$ in the present study, which should be adequate to detect the main features.

\section{Conclusions}

In summary, Fontan surgery resulted in reduced total pulmonary output, possibly owing to the reduced size of the pulmonary arteries. The in-plane velocity analysis showed that the circular flow pattern in the $\mathrm{BC}$ group caused by the two venous flows meeting at the bicaval connection caused higher shear stresses near the wall of the vessels than in the normal volunteers. The disorder caused by the direct connection of the right atrium to the RPA caused an increase in some shear stresses over the control group, but not higher than those found in the $\mathrm{BC}$ group. In comparing the two types of Fontan procedures, the quantity of blood flow and the energy losses associated with the flow investigated in previous models are important in assessing the immediate success of the procedure, whereas the shear stresses examined in this study may influence long-term outcomes of the procedure. The type of data obtained with this method could be used to complement clinical findings and modeling results and to determine the relative advantages and disadvantages of different types of connections.

\section{REFERENCES}

1. Leblanc JG, Williams WG. The operative and post operative management of congenital heart disease. Mount Kisco [NY]. Futura; 1993.

2. Rao PS. Tricuspid atresia, 2nd ed. Mount Kisco [NY] Futura; 1992

3. O'Donnell M. NMR blood flow imaging using multiecho, phase contrast sequences. Med Phys 1985;12:59-64.

4. Naylor GL, Firmin DN, Longmore DB. Blood flow imaging by cine magnetic resonance. J Comput Assist Tomogr 1986; 10:715-22.

5. Pelc NJ, Herfkens RJ, Shimakawa A, Enzmann DR. Phase contrast cine magnetic resonance imaging. Magn Reson Q 1991;7:229-54.

6. Morgan VL, Price RR, Lorenz CH. Application of linear optimization techniques to MRI phase contrast blood flow measurements. Magn Reson Imaging 1996;14:1043-51.

7. Fung YC. Biodynamics circulation. New York. SpringerVerlag; 1984.

8. Craig JJ. Introduction to robotics mechanics and control. 2nd ed. Reading [MA]. Addison-Wesley; 1989.

9. Low HT, Chew YT, Lee CN. Flow studies on atriopulmonary and cavopulmonary connections of the Fontan operations for congenital heart defects. J Biomed Eng 1993;15: 303-7.

10. Van Heasdonck JM, Mertens L, Sizaire R, Montas G, Purnode B, Daenen W, et al. Comparison by computer numeric modeling of energy losses in different Fontan connections. Circulation 1995;92(9 Suppl):II322-6.

11. Rossitti S, Frangos J, Girard PR, Bevan J. Regulation of vasculature tone. Can J Physiol Pharmacol 1995;73:544-50.

12. Davies PF, Dewey CF Jr, Bussolari SR, Gordon EJ, Gimbrone MA Jr. Influence of hemodynamic forces on vascular endothelial function. J Clin Invest 1984;73:1121-9.

13. Fry DL. Acute vascular endothelial changes associated with increased blood velocity gradients. Circ Res 1968;22: 165-97.

14. Ziegler T, Alexander RW, Nerem RM. An endothelial cell-smooth muscle cell model for use in the investigation of flow effects on vascular biology. Ann Biomed Eng 1995;23: 216-25.

15. Rubanyi GM, Romero JC, Vanhoutte PM. Flow-induced release of endothelium-derived relaxing factor. Am J Physiol 1986;250:H1145-9.

16. Ohno M, Gibbons GH, Dzau VJ, Cooke JP. Shear stress elevates endothelial cGMP: role of a potassium channel and G protein coupling. Circulation 1993;88:193-7.

17. Snow HM, McAuliffe SJ, Moors JA, Brownlie R. The relationship between blood flow in the iliac artery of the anaes- 
thetized dog: the role of endothelium-derived relaxing factor and shear stress. Exp Physiol 1994;79:635-45.

18. Melkumyants AM, Balashov SA, Kartamyshev SP. Anticonstrictor effect of endothelium sensitivity to shear stress. Pflugers Arch 1994;427:264-9.

19. Masuda H, Kawamura K, Sugiyama T, Kamiya A. Effects of endothelial denudation in flow-induced arterial dilatation. Front Med Biol Eng 1993;5:57-62.

20. Grabowski EF, Lam FP. Endothelial cell function, including tissue factor expression, under flow conditions. Thromb Haemost 1995;74:123-8.

21. Wu MH, Kouchi Y, Onuki Y, Shi Q, Yoshida H, Kaplan S, et al. Effect of differential shear stress on platelet aggregation, surface thrombosis, and endothelialization of bilateral carotid femoral grafts in the dog. J Vasc Surg 1995;22:382-90.

22. Paz R, Mohiaddin RH, Longmore DB. Magnetic resonance assessment of the pulmonary arterial trunk anatomy, flow, pulsatility and distensibility. Eur Heart J 1993;14:1524-30.

23. Fogel MA, Donofrio MT, Ramaciotti C, Hubbard AM, Weinberg PM. Magnetic resonance and echocardiographic imaging of pulmonary artery size throughout stages of Fontan reconstruction. Circulation 1994;90:2927-36.
24. Penny DJ, Pawade A, Wilkinson JL, Karl TR. Pulmonary artery size after bi-directional cavopulmonary connexion [abstract]. 67th Scientific Session of the American Heart Association Abstract Book 1994:521.

25. Fontan F, Fernandez G, Costa F, Costa F, Naftel DC, Tritto F, et al. The size of the pulmonary arteries and the results of the Fontan operation. J Thorac Cardiovasc Surg 1989;98:71124.

26. Senzaki H. Reconsideration of criteria for the Fontan operation: influence of pulmonary artery size on postoperative hemodynamics of the Fontan operation. Circulation 1994;89: 1196-202.

27. de Leval MR, Dubini G, Migliavacca F, Jalali H, Camporini $\mathrm{G}$, Redington A, et al. Use of computational fluid dynamics in the design of surgical procedures: application to the study of competitive flows in cavopulmonary connections. J Thorac Cardiovasc Surg 1996;111:502-13.

28. Sharma S, Goudy S, Walker P, Panchal S, Ensley A, Kanter $\mathrm{K}$, et al. In vitro experiments for determination of optimal geometry of total cavopulmonary connection for surgical repair of children with functional single ventricle. J Am Coll Cardiol 1996;27:1264-9. 